\title{
SOME APPLICATIONS OF MODULE THEORY TO FUNCTOR CATEGORIES
}

\author{
BY BARRY MITCHELL'
}

Introduction. The notion of an additive category was abstracted from the example of all modules over a ring, a very large category. However with tongue firmly in cheek, one can define a ring with identity (all rings will have identity) as an additive category with just one object. Years ago, the notion of a Morita context was expounded with a certain amount of labour. A Morita context turned out to be an additive category with two objects. It is not inconceivable, then, that someday additive categories with three objects will emerge, the jump from three to infinity will be made, and additive categories will be rediscovered from the point of view of the small examples instead of the big ones. (Heaven knows what they will be called.)

I wish to indicate how the observation that a ring $R$ is an additive category with one object can be used for purposes other than to boggle the student of algebra. First, an $R$-module, from this point of view, is just an additive functor from $R$ to the category $\mathrm{Ab}$ of abelian groups, and an $R$-module homomorphism is a natural transformation between two such functors. Thus, if $\mathcal{C}$ is a small additive category, or what we shall refer to more briefly as a ringoid, then a $\mathcal{C}$-module is a covariant additive functor $M: \mathcal{C} \rightarrow \mathrm{Ab}$, and the category of all such is denoted Mod $\mathcal{C}$. (Actually what we have defined is a

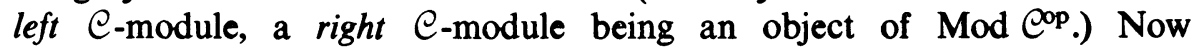
frequently, when such a category arises in the literature, it is pointed out that it is an abelian category, that it has exact direct limits, that it has a set of generators, that it has enough projectives and the injectives, and so on. What needs to be stressed is that there is virtually nothing which one can do in categories of modules over (not necessarily commutative) rings, which can't be done in categories of modules over ringoids.

First, let us consider the building block of the category Mod $R$, namely $R$ considered as a module over itself. In the more general situation, there is a whole family of building blocks, one for each object of $\mathcal{C}$, namely the representable modules (functors) $\mathcal{C}(p$,$) . The additive Yoneda lemma states$ that there is an isomorphism of abelian groups

$$
\operatorname{Hom}_{e}(\mathcal{C}(p,), M) \simeq M(p)
$$

which is natural not only as functors of the $\mathcal{C}$-module $M$, but also as functors of the variable $p$. What is being generalized here, of course, is the familiar natural isomorphism $\operatorname{Hom}_{R}(R, M) \simeq M$ of left $R$-modules. The

An expanded version of an Invited Address delivered at the Annual Meeting of the American Mathematical Society in San Francisco, California, on January 18, 1974; received by the editors November 1, 1977.

AMS (MOS) subject classifications (1970). Primary 18E10, 18H15, 18G20, 13A60, $18 \mathrm{G} 20$.

' Partially supported by NSF Grant 77-03646.

- American Mathematical Society 1978 
isomorphism (1) yields immediately three facts about the modules $\mathcal{C}(p$,$) ;$ they are projective, they are small (that is, homing with them commutes with coproducts), and as a family they generate. In fact, one can show that the existence of a family of objects with these three properties in an abelian category with coproducts actually characterizes categories of the form Mod ${ }^{2}$.

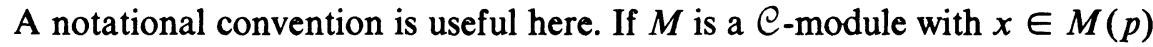
and $\lambda \in \mathcal{C}(p, q)$, then we denote the element $M(\lambda)(x)$ of $M(q)$ simply by $\lambda x$. This is, of course, what one does in module theory over a ring, and one need only keep in mind that in the more general situation every element $x \in M$ comes with an object $p$ attached to it, and that scalar multiplication $\lambda x$ makes sense only when the domain of $\lambda$ is $p$. With this convention in mind, one rarely has anything to change in generalizing definitions and facts concerning modules over rings to modules over ringoids. For example, a family of elements $x_{i} \in M\left(p_{i}\right)$ is a family of generators for $M$ if every element $y \in$ $M(q)$ can be written as $y=\Sigma \lambda_{i} x_{i}$ for some $\lambda_{i} \in \mathcal{C}\left(p_{i}, q\right)$. The family is independent if $\Sigma \lambda_{i} x_{i}=0 \Rightarrow \lambda_{i}=0$ for all $i$, and is a basis if it is andependent family of generators. Equivalently, if

$$
\bigoplus_{i} \mathcal{C}\left(p_{i},\right) \stackrel{\varepsilon}{\rightarrow} M
$$

is the morphism determined by the $x_{i}$ in view of (1), then $\left\{x_{i}\right\}$ is a family of generators if $\varepsilon$ is an epimorphism, independent if $\varepsilon$ is a monomorphism, and a basis if $\varepsilon$ is an isomorphism. A module is free if it has a basis, or equivalently, if it is isomorphic to a coproduct of representables. The notions of finitely generated and finitely presented then have their usual meanings.

A left ideal of $\mathcal{C}$ is a submodule of a representable $\mathcal{C}(p$,$) , a right ideal is a$ submodule of $\mathcal{C}(, p)$ and a (two sided) ideal is a subfunctor of the two variable functor $\mathcal{C}($,$) . If I$ is a two sided ideal, then one can form the quotient ringoid $\mathrm{C} / I$ in the obvious way. Then, just as in ordinary ring theory, once one has defined a property for modules, one says that the ringoid has that property if each of its representables $\mathcal{C}(p$,$) has the property.$ For example, $\mathcal{C}$ is noetherian (artinian) if each $\mathcal{C}(p$,$) has the acc (dcc) on$ submodules.

Granting that generalization from rings to ringoids is automatic, one might (and should) ask, why do it? Certainly there would not be much interest if all that is involved is a series of exercises in a basic course in ring theory. In order to justify generalization of this type, perhaps at least one of the following criteria should be satisfied.

(1) Proofs of known theorems are simplified.

(2) Two or more theorems are combined in one.

(3) New examples are embraced by an old theory.

(4) An open problem is solved.

In what follows, I shall attempt to show that all of these conditions have been met.

1. Projective dimension. Regarding an $R$-module as an additive functor $R \rightarrow \mathrm{Ab}$, one can consider generalizing the range as well as the domain, replacing $\mathrm{Ab}$ by a more general abelian category. For example, an additive 
functor $R \rightarrow \operatorname{Mod} S$ is just an $R, S$-bimodule. Actually, most of our basic lemmas on projective dimension are valid in the context of abelian categories, and we shall state them in this generality. Proofs may be found in [24].

The projective dimension of an object $A$ in an abelian category $\mathcal{Q}$ is defined as

$$
\operatorname{pd} A=\sup \left\{k \mid \operatorname{Ext}^{k}(A,) \neq 0\right\} .
$$

Here we don't even require $\mathbb{Q}$ to have enough projectives, since we can use Yoneda's definition of Ext in terms of exact sequences. Of course, if $\mathbb{Q}$ has enough projectives, then $\mathrm{pd} A$ can be defined alternatively as the shortest length (or infinity) of a projective resolution of $A$. The global dimension of $\mathbb{Q}$ $(\mathrm{gl} \operatorname{dim} \mathcal{Q})$ is the sup of the projective dimensions of its objects. The long exact Ext sequence induced by a short exact sequence

$$
0 \rightarrow C \rightarrow B \rightarrow A \rightarrow 0
$$

in $\mathscr{Q}$ yields immediately:

LEMMA 1.1.

$$
\begin{aligned}
& \operatorname{pd} B=\operatorname{pd} C \Rightarrow \operatorname{pd} A \leqslant 1+\operatorname{pd} C, \\
& \operatorname{pd} B<\operatorname{pd} C \Rightarrow \operatorname{pd} A=1+\operatorname{pd} C, \\
& \operatorname{pd} B>\operatorname{pd} C \Rightarrow \operatorname{pd} A=\operatorname{pd} B .
\end{aligned}
$$

If $\eta: F \rightarrow G$ is a natural transformation between functors $F, G: \mathbb{Q} \rightarrow \mathscr{B}$, then we say that $\eta$ is a pointwise coretraction if for each object $A \in \mathbb{Q}$, there is a morphism $\mu_{A}: G A \rightarrow F A$ such that $\mu_{A} \eta_{A}=$ identity. (We don't require $\mu$ to be natural.)

Lemma 1.2. Consider exact functors $T: \mathscr{B} \rightarrow \mathbb{Q}$ and $S: \mathbb{Q} \rightarrow \mathscr{B}$ between abelian categories.

(a) If there is a pointwise coretraction $\eta: 1_{\mathfrak{Q}} \rightarrow T S$, then $\mathrm{pd} S A \geqslant \operatorname{pd} A$ for all $A \in \mathbb{Q}$. Hence $\mathrm{gl} \operatorname{dim} \mathscr{B} \geqslant \mathrm{gl} \operatorname{dim} Q$.

(b) If $S$ is left adjoint to $T$, then $\mathrm{pd} S A \leqslant \mathrm{pd} A$ for all $A \in \mathbb{Q}$.

Both parts of Lemma 1.2 apply in the following situation. Consider a small category $\mathbf{C}$ and an object $p \in \mathbf{C}$. Then we have the $p$ th evaluation functor $T_{p}$ : $\mathbb{Q}^{\mathrm{C}} \rightarrow \mathbb{Q}$, defined by $T_{p}(D)=D(p)$. If $\mathbb{Q}$ has coproducts, then the left adjoint of $T_{p}$ is the functor $S_{p}$ defined by

$$
S_{p}(A)(q)=\underset{\mathbf{C}(p, q)}{\bigoplus} A .
$$

The adjunction morphism

$$
\eta_{A}: A \rightarrow T_{p} S_{p}(A)=\underset{\mathrm{C}(p, p)}{\bigoplus} A
$$

is just the coproduct injection corresponding to $1_{p}$. Now if $\mathscr{Q}$ is abelian with exact coproducts, then $S_{p}$ is exact, and since $T_{p}$ is exact in any case, Lemma 1.2 yields:

Corollary 1.3. If $Q$ is abelian with exact coproducts, then $\operatorname{pd} S_{p} A=\operatorname{pd} A$ for all $A \in \mathbb{Q}$ and $p \in \mathbf{C}$.

Part (a) of Lemma 1.2 applies, of course, if $T S$ is the identity functor, that 
is, if $\mathcal{Q}$ is a retract of $\mathscr{B}$ via exact functors. Now if $\mathbf{C}$ is a retract of $\mathbf{D}$ in Cat, then $\mathbb{Q}^{C}$ is a retract of $\mathbb{Q}^{D}$ via exact functors, and so we obtain:

Corollary 1.4. Let $\mathbf{C}$ be a retract of $\mathbf{D}$ and let $F: D \rightarrow C$ be a retraction. If $\mathbb{Q}$ is an abelian category and $D \in \mathbb{Q}^{\mathrm{C}}$, then $\mathrm{pd} D F>\operatorname{pd} D$. Consequently, $\mathrm{gl} \operatorname{dim} \mathbb{Q}^{\mathrm{D}}>\mathrm{gl} \operatorname{dim} \mathbb{Q}^{\mathbf{C}}$.

If 1 denotes the one morphism category, then 1 is a retract of any nonempty category $\mathbf{D}$. Consequently:

Corollaky 1.5. gl $\operatorname{dim} \mathbb{Q}^{\mathrm{D}}>\mathrm{gl} \operatorname{dim} Q$ for any nonempty, small category $\mathbf{D}$ and any abelian category $\mathbb{Q}$.

Part (b) of Lemma 1.2 is an immediate consequence of the natural isomorphism

$$
\operatorname{Ext}_{\mathscr{B}}^{n}(S A, B) \simeq \operatorname{Ext}_{\mathbb{Q}}^{n}(A, T B)
$$

which is valid whenever $S$ is an exact left adjoint for an exact functor $T$. This isomorphism can also be used to prove the following lemma.

LEMMA 1.6. If $Q$ is an abelian category with exact coproducts, then

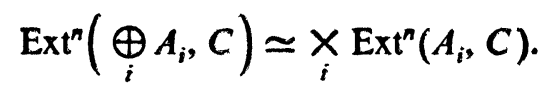

Hence $\mathrm{pd} \bigoplus_{i} A_{i}=\sup \left\{\mathrm{pd} A_{i}\right\}$.

So far we have no means for exhibiting abelian categories of arbitrarily large global dimension. The following lemma, which puts us in a situation similar to, but somewhat more complicated than that of Lemma 1.2, part (a), fulfills this purpose.

Lemma 1.7 (The dimension RaIsing lemma). Consider exact functors $T_{0}, T_{1}$ : $\mathscr{B} \rightarrow \mathbb{Q}$ and $S: \mathbb{Q} \rightarrow \mathscr{B}$, and suppose there is a pointwise coretraction $\eta:$ $1_{A} \rightarrow T_{1} S$ and a natural transformation $\varphi: T_{0} \rightarrow T_{1}$ with $\varphi_{S A}=0$ for all $A \in \mathbb{Q}$. Consider also an exact sequence

$$
0 \rightarrow S A \stackrel{\mu}{\rightarrow} B \rightarrow B^{\prime} \rightarrow 0
$$

in $\mathscr{B}$, and suppose there is a morphism $\tau$ in $\mathscr{Q}$ making the diagram

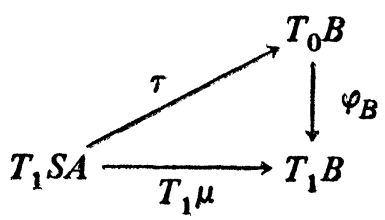

commutative. Then $\mathrm{pd} B^{\prime}>1+\mathrm{pd} A$.

The proof is much shorter than the statement. It may be illuminating to illustrate the lemma with the simplest situation to which it applies. Let 2 be the category $0 \stackrel{x}{\rightarrow} 1$, the totally ordered set of two elements. Then the functor category $\mathbb{Q}$ is the category whose objects are the morphisms of $\mathbb{Q}$ and whose morphisms are commutative squares. We have two evaluation functors $T_{0}, T_{1}: \mathbb{Q}^{2} \rightarrow \mathbb{Q}$, and $x$ induces a natural transformation $\varphi: T_{0} \rightarrow T_{1}$. If $A \in \mathcal{Q}$, then we have the diagram in $\mathcal{Q}$ 


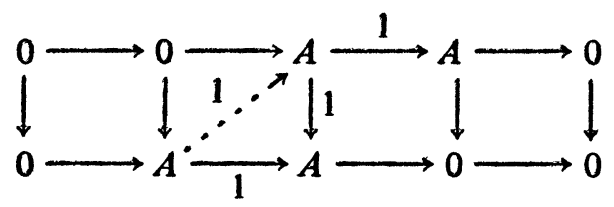

which may be considered as an exact sequence

$$
0 \rightarrow S_{1} A \rightarrow B \rightarrow B^{\prime} \rightarrow 0
$$

in $\mathbb{Q}^{2}$. Note that $T_{1} S_{1}$ is the identity, and that $\varphi_{S_{1} A}=0$. Therefore if we take $\tau=1_{A}$, then all the conditions of the dimension raising lemma are satisfied, and we conclude that $\mathrm{pd} B^{\prime}>1+\mathrm{pd} A$. Consequently gl $\operatorname{dim} \mathbb{Q}^{2} \geqslant 1+\mathrm{gl}$ $\operatorname{dim} \mathbb{Q}$.

Another simple application of the dimension raising lemma shows that if $\mathbf{N}$ is the free monoid on one generator (so that $\mathbb{Q}^{\mathrm{N}}$ is the category of endomorphisms in $\mathcal{Q}$ ), then $\mathrm{gl} \operatorname{dim} \mathbb{Q}^{\mathrm{N}} \geqslant 1+\mathrm{gl} \operatorname{dim} \mathcal{Q}$. Similarly, if $\mathbf{Z}$ denotes the free (multiplicative) group on one generator, then $\mathbb{Q}^{\mathbf{z}}$ is the category of automorphisms in $\mathcal{Q}$, and one uses the dimension raising lemma again to see that $\mathrm{gl} \operatorname{dim} \mathbb{Q}^{\mathbf{Z}}>1+\mathrm{gl} \operatorname{dim} \mathbb{Q}$.

2. The generalized syzygy theorem. If $\mathcal{C}$ is a ringoid, then the global dimension of Mod $\mathcal{C}$ is denoted simply by $\mathrm{gl} \operatorname{dim} \mathcal{C}$. The Hilbert syzygy theorem states that $\mathrm{gl} \operatorname{dim} R[X]=1+\mathrm{gl} \operatorname{dim} R$. However there are other theorems which say that if you do something to a ring, you raise global dimension by one. This happens, for example, if you pass to the ring of $n \times n$ triangular matrices over $R, n \geqslant 2$, or the monoid (group) ring $R G$ where $G$ is a free monoid (group) on at least one generator. In this section we shall show how all these theorems can be combined in one.

Let $\mathbf{C}$ be the free category generated by a directed graph. Thus the objects of $\mathbf{C}$ are the vertices of the graph, and the morphisms are the "paths" in the graph. The functor category $\mathbb{Q}^{C}$ is the same as the category of diagrams in $\mathscr{Q}$ over the graph. If $D \in \mathbb{Q}^{C}$, it is not difficult to write down an exact sequence in $Q^{\mathrm{C}}$

$$
0 \rightarrow \underset{a}{\bigoplus} S_{\operatorname{cod} a} D(\operatorname{dom} a) \rightarrow \underset{p \in \mathbf{C}}{\bigoplus_{p}} S_{p}(D(p)) \rightarrow D \rightarrow 0
$$

where $a$ runs through the arrows of the graph, and dom and cod denote respectively domain and codomain (range). Actually the sequence is still valid if $\mathbf{C}$, instead of being a free category, is some category of fractions of such. This remark is necessary if we want to include the example of the free group mentioned above. Now if $Q$ has exact coproducts, then by Corollary 1.3 and Lemma 1.6, the left and middle terms in the sequence (1) have projective dimension $<\sup _{p} \mathrm{pd} D(p)$. Therefore by Lemma 1.1 we obtain

$$
\text { pd } D<1+\operatorname{suppd}_{p} D(p) \text {. }
$$

Consequently

$$
\text { gl } \operatorname{dim} \mathbb{Q}^{\mathrm{C}}<1+\mathrm{gl} \operatorname{dim} \mathcal{Q} .
$$

Now let us suppose that the morphisms (paths) in the free category which are inverted so as to obtain $\mathbf{C}$ are all of length one (that is, arrows of the 
original graph). Such a category $\mathbf{C}$ is called a bridge category. It is not difficult to see that if $\mathbf{C}$ is a bridge category which is not equivalent to a discrete category, then $\mathbf{C}$ contains at least one of $2, \mathbf{N}$, or $\mathbf{Z}$ as a retract in Cat. But the dimension raising lemma gave us $\mathrm{gl} \operatorname{dim} \mathbb{Q}^{\mathbf{C}} \geqslant 1+\mathrm{gl} \operatorname{dim} \mathscr{Q}$ in each of these three cases. Therefore the same holds for a general bridge category by Corollary 1.4. Combining with (3), this yields:

THEOREM 2.1 (GENERALIZED SYZYGY THEOREM). Let $\mathbb{Q}$ be an abelian category with exact coproducts, and let $\mathrm{C}$ be a bridge category which is not equivalent to a discrete category. Then

$$
\text { gl } \operatorname{dim} Q^{C}=1+\text { gl } \operatorname{dim} Q .
$$

REMARK. If $\mathbf{D}$ is a category of fractions of a free category and $\mathbf{C}$ is a category presented as a quotient of $\mathbf{D}$ modulo some congruence relation, then one can form the sequence (1) except that in place of the 0 on the left end there is a coproduct indexed by relations generating the congruence relation, and one can add a subsequent term indexed by the "relations among relations." This sequence is a generalization of one given by Lyndon [22] relative to group cohomology, and can be found in [24, p. 104].

We shall now show how one derives statements about rings from a theorem such as the above. If $\mathbf{C}$ is a small category and $R$ is a ring, we can form the ringoid $R \mathrm{C}$ whose objects are those of $\mathrm{C}$, where $R \mathrm{C}(p, q)$ is the free $R$-module on the set $\mathbf{C}(p, q)$. Composition is defined by

$$
\left(\sum_{\beta} r_{\beta}^{\prime} \beta\right)\left(\sum_{\alpha} r_{\alpha} \alpha\right)=\sum_{\gamma}\left(\sum_{\gamma=\beta \alpha} r_{\beta}^{\prime} r_{\alpha}\right) \gamma \text {. }
$$

If $\mathbf{C}$ is a monoid (category with just one object), then $R \mathbf{C}$ is, of course, the usual monoid ring with coefficients in $R$. Now it is easy to write down an isomorphism of categories

$$
\operatorname{Mod} R \mathbf{C} \simeq(\operatorname{Mod} R)^{\mathrm{C}} .
$$

The classical syzygy theorem now follows since $R \mathbf{N}$ is just the polynomial ring $R[X]$, more generally taking $G$ to be a free monoid on at least one generator, we get the theorem of Hochschild [13]: $\mathrm{gl} \operatorname{dim} R G=1+\mathrm{gl} \operatorname{dim} R$. Similarly, since a free group is just a free monoid with all generators inverted, we obtain the same result for the free group ring.

To see how to get the theorem on triangular matrix rings, define first two ringoids $C$ and $\mathscr{D}$ to be Morita equivalent if their module categories are equivalent. If $\mathcal{C}$ is a ringoid whose set $|\mathcal{C}|$ of objects is finite, we can form the ring $[\mathcal{C}]$ of $|\mathcal{C}| \times|\mathcal{C}|$ matrices of the form $\left[\alpha_{p q}\right]$ where $\alpha_{p q} \in \mathcal{C}(q, p)$. Using the fact that $\bigoplus_{p} \mathcal{C}(p$,$) is a small projective generator for Mod \mathcal{C}$, one sees that $\mathcal{C}$ and $[\mathcal{C}$ ] are Morita equivalent. In particular if $\mathbf{C}$ is a finite poset, then considering $\mathbf{C}$ as a category, $[R \mathbf{C}]$ is just the ring of $|\mathbf{C}| \times|\mathbf{C}|$ matrices of the form $\left[r_{p q}\right]$ with $r_{p q} \in R$ and $r_{p q}=0$ if $p \neq q$. Taking $\mathbf{C}$ to be the totally ordered set with $n$ elements (which is a free category), we see that $[R C]$ is the ring of $n \times n$ triangular matrices.

3. Maschke's theorem. We consider briefly another classical theorem which 
turns out to be a theorem about abelian categories. If $\mathbb{Q}$ is an additive category, then the center of $\mathcal{Q}$, denoted $C(\mathbb{Q})$, is the ring of endomorphisms of the identity functor on $\mathcal{Q}$. If $\mathcal{C}$ is a ringoid, then $C(\operatorname{Mod} \mathcal{C}) \simeq C(\mathcal{C})$. The center of a ring is, needless to say, isomorphic to the subring of all elements which commute with every element.

THEOREM 3.1. Let $\mathbb{Q}$ be an abelian category, and let $G$ be a finite group. If the order of $G$ is invertible in $C(Q)$, then

$$
\text { gl } \operatorname{dim} \mathbb{Q}^{G}=\mathrm{gl} \operatorname{dim} \mathbb{Q} .
$$

Otherwise $\mathrm{gl} \operatorname{dim} \mathbb{Q}^{G}=\infty$.

Corollary 3.2 (MASCHKE). If $G$ is a finite group and $K$ is a field whose characteristic does not divide the order of $G$, then $K G$ is semisimple.

The theorem is proved in [23]. The statement about infinite global dimension is another application of the dimension raising lemma.

4. Partially ordered sets. Let $C$ be a poset and let $L$ be a subcategory. If there is a retraction $F: \mathbf{C} \rightarrow \mathbf{L}$, then it is obvious that $\mathbf{L}$ must be full. On the other hand suppose that $\mathbf{L}$ is a full subcategory, and suppose further that $\mathbf{L}$ is a complete lattice. Then we can define $F: \mathbf{C} \rightarrow \mathbf{L}$ by letting $F(p)$ be the inf of all members of $\mathbf{L}$ greater than or equal to $p$. The map $F$ is order preserving, and by fullness restricts to the identity on $\mathbf{L}$. In this case by Corollary 1.4 we obtain

$$
\text { gl } \operatorname{dim} Q^{\mathbf{C}} \geqslant \mathrm{gl} \operatorname{dim} \mathbb{Q}^{\mathbf{L}}
$$

for any abelian category $\mathcal{Q}$. Now we have seen that

$$
\text { gl } \operatorname{dim} \mathbb{Q}^{2}=1+\mathrm{gl} \operatorname{dim} Q,
$$

and by induction we obtain

$$
\text { gl } \operatorname{dim} \mathbb{Q}^{2^{n}}=n+\mathrm{gl} \operatorname{dim} \mathcal{Q} .
$$

Since $2^{n}$ is a complete lattice, we then see that

$$
\text { gl } \operatorname{dim} \mathbb{Q}^{C} \geqslant n+\mathrm{gl} \operatorname{dim} Q
$$

for any poset containing $2^{n}$ as a full subset.

A poset $\mathbf{C}$ is discrete if $p \leqslant q \Rightarrow p=q$. In this case $\mathbb{Q}^{\mathbf{C}}$ is just a product of copies of $\mathcal{Q}$. On the other hand if $\mathbf{C}$ is not discrete, then $\mathbf{C}$ contains $\mathbf{2}$ as a (necessarily full) subset. This gives:

Proposition 4.1. If $\mathbf{C}$ is a discrete poset and $Q$ is any abelian category, then

$$
\text { gl } \operatorname{dim} \mathbb{Q}^{\mathbf{C}}=\mathrm{gl} \operatorname{dim} \mathbb{Q} \text {. }
$$

On the other hand if $\mathbf{C}$ is not discrete, then

$$
\text { gl } \operatorname{dim} \mathbb{Q}^{\mathbf{C}} \geqslant 1+\mathrm{gl} \operatorname{dim} Q .
$$

If $p \leqslant q$ in a poset, then the full subset $\{v \mid p \leq v \leq q\}$ is called a muscle in C. Clearly $\mathbf{C}$ contains $2 \times 2$ as a full subset if and only if some muscle is not totally ordered. In this case we shall say that $\mathbf{C}$ contains a square. The Krull dimension of a poset is the sup of the lengths of its chains. A poset $\mathbf{C}$ is free as a category if and only if all of its muscles are finite chains, or equivalently, if 
and only if its muscles have finite Krull dimension and $\mathbf{C}$ does not contain a square. Combining this with the generalized syzygy theorem, we obtain:

THEOREM 4.2. If $\mathbf{C}$ is a poset whose muscles have finite Krull dimension and if $\mathrm{C}$ does not contain a square, then

$$
\mathrm{gl} \operatorname{dim} \mathbb{Q}^{\mathbf{C}} \leqslant 1+\mathrm{gl} \operatorname{dim} Q
$$

for all abelian categories $\mathbb{Q}$ with exact coproducts. On the other hand if $\mathbf{C}$ is any poset containing a square, then

for all abelian categories $\mathbb{Q}$.

$$
\text { gl } \operatorname{dim} Q^{C} \geqslant 2+\operatorname{gl} \operatorname{dim} Q
$$

REMARK. If $\mathbf{C}$ is a well ordered set and $K$ is a field, then every left ideal in $K \mathbf{C}$ is representable. Thus $K \mathrm{C}$ is hereditary and so $\mathrm{gl} \operatorname{dim} K \mathbf{C} \leqslant 1$. On the other hand if $\mathbf{C}$ has ordinal type at least $\omega_{n}+1$ where $\omega_{n}$ denotes the first ordinal of cardinal number $\boldsymbol{\kappa}_{n}$, then using Theorem 5.8 of the following section one can show $\mathrm{gl} \operatorname{dim} K \mathbf{C}^{\text {op }} \geqslant n+2$. But of course no totally ordered set contains a square, and so the assumption on finite Krull dimension cannot be dropped in the first statement of the theorem.

Let us consider the following poset, which we shall denote by $\mathbf{C}_{n}(n \geqslant 2)$ :

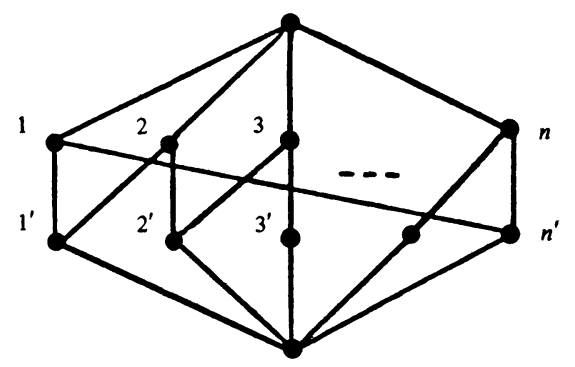

Using the dimension raising lemma, it is not difficult to show that

$$
\text { gl } \operatorname{dim} \mathbb{Q}^{\mathbf{C}_{n}}=3+\mathrm{gl} \operatorname{dim} \mathscr{Q}
$$

for all abelian categories $\mathcal{Q}$. (For $n=3$ this follows since $C_{3}=\mathbf{2} \times \mathbf{2} \times \mathbf{2}$.) But $\mathbf{C}_{n}$ is a finite lattice for $n \geqslant 3$, and consequently

$$
\mathrm{gl} \operatorname{dim} \mathbb{Q}^{\mathbf{C}} \geqslant 3+\mathrm{gl} \operatorname{dim} \mathscr{Q}
$$

for any poset $\mathbf{C}$ containing $\mathbf{C}_{n}$ as a full subset, $n \geqslant 3$. However $\mathbf{C}_{2}$ is not a lattice, and the fact that $\mathbf{C}$ contains it as a full subset does not guarantee that it contains it as a retract. In fact, if $\mathbf{C}_{2}$ is a full subset of $\mathbf{C}$, then it is a retract if and only if there is no element of $\mathbf{C}$ following both $\mathbf{1}^{\prime}$ and $2^{\prime}$ and preceding both 1 and 2 (diagram (1)). We shall say that $C$ contains $a$ crown if it contains $\mathrm{C}_{n}$ as a full subset for some $n \geqslant 2$. with the above additional condition in case $n=2$.

THEOREM 4.3. If $\mathbf{C}$ is a poset whose muscles have finite Krull dimension and if $\mathrm{C}$ does not contain a crown, then

$$
\mathrm{gl} \operatorname{dim} \mathbb{Q}^{\mathbf{C}}<2+\mathrm{gl} \operatorname{dim} \mathbb{Q}
$$

for all abelian categories $\mathbb{Q}$ with exact coproducts. On the other hand if $\mathbf{C}$ is any poset containing a crown, then 
$\mathrm{gl} \operatorname{dim} \mathbb{Q}^{\mathbf{C}} \geqslant 3+\mathrm{gl} \operatorname{dim} \mathbb{Q}$

for all abelian categories $\mathbb{Q}$.

The second statement was essentially proved above. The first statement is more difficult, and is best proved using Theorem 5.6 concerning posets of cohomological dimension one. (See [7, Theorem 15].)

Finally, we remark that there are examples of finite posets $\mathbf{C}$ such that $\mathrm{gl}$ $\operatorname{dim} R \mathbf{C}=3+\mathrm{gl} \operatorname{dim} R$ for some rings $R$, and $\mathrm{gl} \operatorname{dim} R \mathbf{C}=4+\mathrm{gl} \operatorname{dim} R$ for others. Thus there is no sequel to Theorem 4.3.

5. Cohomological dimension. If $\mathbf{C}$ is a small category, then the unique functor $\mathbf{C} \rightarrow \mathbf{1}$ induces a functor $\Delta: \mathbb{Q} \rightarrow \mathbb{Q}^{\mathbf{C}}$ whose right adjoint is denoted $\lim _{\mathbf{C}}: \mathbb{Q}^{\mathbf{C}} \rightarrow \mathbb{Q}$ (lim when there is no danger of confusion), and whose left adjoint is denoted colim ${ }_{\mathrm{C}}$. In particular if $R$ is a ring and $\mathscr{Q}$ is $\operatorname{Mod} R$, then relative to an object $M \in \operatorname{Mod} R \mathbf{C}$ we have

$$
\lim M \simeq \operatorname{Hom}_{R}(R, \lim M) \simeq \operatorname{Hom}_{R C}(\Delta R, M) .
$$

Consequently, if $\lim ^{k}: \operatorname{Mod} R \mathbf{C} \rightarrow \operatorname{Mod} R$ denotes the $k$ th right derived functor of lim, then we see

$$
\lim ^{k} M=\operatorname{Ext}_{R \mathrm{C}}^{k}(\Delta R, M) .
$$

This means that if we define the $R$-cohomological dimension of the small category $\mathbf{C}$ by

$$
\operatorname{cd}_{R} \mathrm{C}=\sup \left\{k \mid \lim ^{k} \neq 0\right\},
$$

then $\mathrm{cd}_{R} \mathrm{C}$ is the same as pd $\Delta R$, the projective dimension of the constant $R$-valued functor considered as an $R \mathrm{C}$-module. When $R=\mathrm{Z}$, the ring of integers, we simply write cd $\mathbf{C}$.

Several observations are immediate.

(1) If $\left\{\mathbf{C}_{i}\right\}$ are the connected components of $\mathbf{C}$, then $\operatorname{Mod} R \mathbf{C}$ is isomorphic to the product of the $\operatorname{Mod} R \mathrm{C}_{i}$, and under the isomorphism $\Delta R$ goes to the tuple of $\Delta R$ 's. This yields

$$
\operatorname{cd}_{R} \mathbf{C}=\sup _{i} \operatorname{cd}_{R} \mathbf{C}_{i} \text {. }
$$

(2) If $\mathbf{C} \rightarrow \mathbf{D}$ is any functor between small categories, then $\Delta R$ goes to $\Delta R$ under the induced functor $\operatorname{Mod} R C \leftarrow \operatorname{Mod} R D$. From Corollary 1.4 it follows that if $\mathbf{C}$ is a retract of $\mathbf{D}$, then $\operatorname{cd}_{R} \mathbf{C} \leqslant \operatorname{cd}_{R} \mathbf{D}$.

(3) If $\hat{\mathbf{C}}$ is the idempotent completion of $\mathbf{C}$, then the inclusion $\mathbf{C} \rightarrow \hat{\mathbf{C}}$ induces an equivalence $\operatorname{Mod} R \hat{\mathbf{C}} \simeq \operatorname{Mod} R \mathrm{C}$. Since $\Delta R$ goes to $\Delta R$, we see that $\operatorname{cd}_{R} \hat{\mathbf{C}}=\operatorname{cd}_{R} \mathbf{C}$.

(4) If there exists a ring homomorphism $R \rightarrow S$, then a projective resolution for $\Delta R$ can be tensored with $S$ over $R$ to obtain a projective resolution for $\Delta S$. It follows that $\mathrm{cd}_{S} \mathrm{C} \leqslant \mathrm{cd}_{R} \mathrm{C}$. In particular we find $\mathrm{cd}_{R} \mathrm{C} \leqslant \mathrm{cdC}$ for all rings $R$. One can show further that if $R \rightarrow S$ is a coretraction as $R$-bimodules, then $\operatorname{cd}_{S} \mathbf{C}=\operatorname{cd}_{R} \mathbf{C}$. This will be the case whenever $S$ is a nonzero $R$-algebra and $R$ is a field.

If $\mathbf{C}$ has an initial object $p$, then $\Delta R$ is just the representable functor $R \mathbf{C}(p$,$) , and consequently \mathrm{cd}_{R} \mathbf{C}=0$. Conversely, we have the following theorem of Laudal [20]. (See also [6].) 
THEOREM 5.1 (LAUDAL). If $\mathbf{C}$ is a connected category in which idempotents split, then $\mathrm{cd} \mathbf{C}=0$ if and only if $\mathbf{C}$ has an initial object.

The assumption on $\mathbf{C}$ is really no restriction, of course, in view of observations (1) and (3) above. Further, if $\mathbf{C}$ is a poset, then the theorem is valid for any coefficient ring $R$. The following lemma shows, however, that arbitrary coefficients cannot be used in general.

LEMMA 5.2. If $G$ is a group and $R$ is a nonzero ring, then $\operatorname{cd}_{R} G=0$ if and only if $G$ is finite and its order is invertible in $R$. If $G$ is finite and its order is not invertible in $R$, then $\mathrm{cd}_{R} G=\infty$.

Now let us consider the cohomological dimension one. Taking $D=\Delta R$ in the inequality (2) of $\$ 2$, we obtain:

Proposition 5.3. If $\mathbf{C}$ is a category of fractions of a free category, then $\mathrm{cd}_{R} \mathbf{C} \leqslant 1$ for any ring $R$.

When $C$ is a torsion free group, the converse of Proposition 5.3 is true, and this is the well-known and difficult theorem of Stallings [28] and Swan [29].

Theorem 5.4 (Stallings, SWAN). If $G$ is a torsion free group and $R$ is any nonzero ring, then $\operatorname{cd}_{R} G \leqslant 1$ if and only if $G$ is free as a group.

Shapiro's lemma asserts that if $H<G$ is a subgroup, then $\operatorname{cd}_{R} H \leqslant \operatorname{cd}_{R} G$. This is another consequence of Lemma $1.2(\mathrm{~b})$, since it is easy to see that the restriction functor $\operatorname{Mod} R G \rightarrow \operatorname{Mod} R H$ has an exact right adjoint. This yields:

Corollary 5.5. If $G$ is any group, then $\mathrm{cd} G \leqslant 1$ if and only if $G$ is a free group.

Let $\mathbf{C}_{n}^{\prime}$ denote the poset $\mathbf{C}_{n}$ without its initial element (diagram (1) of $\$ 4$ ).

Theorem 5.6 (Cheng, Mitchell [7]). Let $\mathbf{C}$ be a poset with dcc and let $R$ be any nonzero ring. Then $\mathrm{cd}_{R} \mathbf{C} \leqslant 1$ if and only if $\mathbf{C}$ does not contain $\mathbf{C}_{n}^{\prime}$ as a retract for any $n \geqslant 2$.

A simple algorithm was given by Cheng [8] for determining when a finite poset has cohomological dimension one. First, if $p<q$ in a finite poset, and if for no $v$ do we have $p<v<q$, then $q$ is called a cover for $p$ and $p$ is a cocover for $q$. An element is superfluous if it has precisely one cocover, or if it is minimal and has precisely one cover. The removal of a superfluous element from a poset may, in the resulting full subset, create new superfluous elements and destroy old ones. We let $E \mathbf{C}$ denote any full subset obtained by iterating as many times as possible the operation of removing a superfluous element. One can show that up to isomorphism, $E \mathrm{C}$ is independent of the way in which superfluous elements are removed. If $\mathbf{C}$ is any poset, we let $\mathbf{C}_{q}$ be the full subset $\{p \mid p \leqslant q\}$.

THEOREM 5.7 (CHENG). Let $\mathbf{C}$ be a poset such that $\mathbf{C}_{q}$ is finite for each $q$. If $R$ is any nonzero ring, then $\mathrm{cd}_{R} \mathbf{C} \leqslant 1$ if and only if $E \mathbf{C}_{q}=1$ for all $q$.

For posets which do not have the dcc we have no conjecture. The 
independence of the ring in Theorem 5.6 breaks down in higher dimensions, for there exists finite posets $\mathbf{C}$ such that $\operatorname{cd}_{R} \mathbf{C}=2$ for some rings $R$ and 3 for others. In fact, one can make the difference $\mathrm{cd}_{R} \mathbf{C}-\mathrm{cd}_{S} \mathbf{C}$ as large as one likes by suitably choosing the finite poset $\mathbf{C}$ and the rings $R$ and $S$. However, for directed sets, the complete story can be told.

THEOREM 5.8 [26]. If $\mathbf{C}^{\mathrm{OP}}$ is a directed set and $R$ is any nonzero ring, then

$$
\mathrm{cd}_{R} \mathbf{C}=n+1
$$

where $\boldsymbol{N}_{n}$ is the smallest cardinal number of a cofinal subset of $\mathbf{C}^{\text {op }}$.

Here we make the conventions that $n+1$ is to be replaced by $\infty$ if $n$ is an infinite ordinal, and $x_{-1}=1$. Thus in the latter case, Theorem 5.8 follows from Theorem 5.1. Notice that Theorem 5.8 serves to show that Theorem 5.6 is not valid without the dcc.

A word on the proof of Theorem 5.8 is perhaps in order. In [27], Osofsky defined the notion of a directed module, and obtained a complete result on the projective dimension of a totally ordered module. Now in keeping with the point of view that most theorems about modules over rings are really theorems about modules over ringoids, Osofsky's theorem is valid for ringoids. With this generality at hand, $\Delta R$ becomes an example of a directed module over $R C$ when $C^{\text {op }}$ is a directed set. This yields easily the totally ordered case of Theorem 5.7. The general case is obtained from the totally ordered case by a trick.

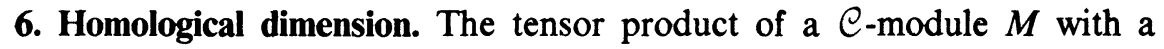
eop-module $N$ is the abelian group defined as

$$
N \bigotimes_{\mathcal{C}} M=\left[\bigoplus_{p} N(p) \underset{\mathbf{Z}}{\otimes} M(p)\right] / K
$$

where $K$ is the subgroup of the numerator generated by elements of the form $n \lambda \otimes m-n \otimes \lambda m$ with $m \in M(p), n \in N(q)$, and $\lambda \in \mathcal{C}(p, q)$. The usual tensor-hom adjointness is readily established. It is also easy to exhibit an isomorphism

$$
N \underset{e}{\bigotimes} C(p,) \simeq N(p)
$$

which is natural in both $N$ and $p$. This is the obvious extension of the familiar isomorphism $N \otimes_{R} R \simeq N$ of right $R$-modules. The usual notions of flatness and purity make sense in this generality, and the standard tools relating to them are available. For example, D. Lazard's theorem [21] characterizing flat modules as direct limits of projective modules is valid, and so the notion of flatness is a Morita invariant. Likewise for purity.

Now consider the left adjoint colim: $\operatorname{Mod} R \mathbf{C} \rightarrow \operatorname{Mod} R$ of the functor $\Delta$ : $\operatorname{Mod} R \rightarrow \operatorname{Mod} R C$. Using tensor-hom adjointness, colim is easily seen to be given by

$$
\text { colim } M=\Delta R \bigotimes_{R \mathrm{C}} M \text {. }
$$

Thus if colim $_{k}$ denotes the $k$ th left derived functor of colim, then we see

$$
\operatorname{colim}_{k} M=\operatorname{Tor}_{k}^{R \mathrm{C}}(\Delta R, M) \text {. }
$$


This means that if we define the $R$-homological dimension of the small category $\mathbf{C}$ by

$$
\operatorname{hd}_{R} C=\sup \left\{k \mid \operatorname{colim}_{k} \neq 0\right\},
$$

then $\mathrm{hd}_{R} C$ is the same as wd $\Delta R$, the weak (or flat) dimension of the constant $R$-valued functor considered as an $(R \mathbf{C})^{\mathrm{op}}$-module. Again for $R=\mathbf{Z}$, we write hd $\mathbf{C}$. The observations made on cohomological dimension at the beginning of the previous section are also valid for homological dimension.

A category $\mathbf{C}$ is filtered if:

(1) For every pair of objects $p, q$, there is an object $r$ such that $\mathbf{C}(p, r)$ and $\mathrm{C}(q, r)$ are nonempty.

(2) For every pair of morphisms $\alpha, \beta \in \mathbf{C}(p, q)$, there is a morphism $\gamma$ such that $\gamma \alpha=\gamma \beta$.

When $\mathbf{C}$ is a poset, (2) is redundant, and (1) just says that $\mathbf{C}$ is directed. In this case we know, of course, that the colimit functor (usually called the direct limit functor) is exact, and it is easy to see that this is true more generally for filtered categories. Consequently it is true for categories all of whose components are filtered, and so for such categories we have $\mathrm{hd}_{R} \mathbf{C}=0$. A conjecture which stood for some time was that when $R=\mathbf{Z}$ the converse is true. Before giving the counter-example, we shall state the correct theorem. If $\mathbf{C}$ is a small category, then aff $\mathbf{C}$ denotes the subcategory (nonadditive) of $\mathbf{Z C}$ consisting of those morphisms whose integer coefficients sum to 1 .

THEOREM 6.1. hd $\mathbf{C}=0$ if and only if aff $\mathbf{C}$ has filtered components.

This theorem appeared in Isbell and Mitchell [15] and is essentially a reworking of an earlier theorem of Isbell. It is proved by observing that hd $\mathbf{C}=0$ means $\Delta \mathbf{Z}$ is flat as a $\mathbf{Z C}^{\text {op }}$-module, so that consequently the monomorphism is pure in a short resolution for $\Delta \mathbf{Z}$. One then invokes a well-known criterion for purity in terms of generators and relations. Note that the theorem says that the above conjecture is true if $\mathbf{C}$ is a poset, since in this case aff $\mathbf{C}=\mathbf{C}$. Also, using the theorem one can show that the conjecture is true if $\mathbf{C}$ has a weak terminal object [15]. Such considerations lead the second author above to conjecture that the category $\boldsymbol{\Delta}_{\text {face }}$ of all finite ordinals and order preserving injections is a counterexample to the original conjecture. It is clearly not filtered since all of its morphisms are monomorphisms. An ingenious argument of Isbell [14] showed that, in fact, aff $\Delta_{\text {face }}$ is filtered.

Besides the above theorem, not too much seems to be known about homological dimension. In particular, I don't believe there are any theorems on homological dimension one. Locally free groups are easily seen to have homological dimension one, and one could conjecture that the converse is true. However I don't know if the problem has been looked at. There is, nevertheless, a theorem relating homological dimension to cohomological dimension.

TheOREM 6.2 (LATCh, Mitchell [19]). If the cardinal number of the set of morphisms of $\mathrm{C}$ is $\aleph_{n}$, then

$$
\operatorname{hd}_{R} \mathbf{C} \leqslant \operatorname{cd}_{R^{\circ p}} C^{\text {op }} \leqslant n+1+\operatorname{hd}_{R} C .
$$


The first inequality follows from the fact that the weak dimension of any module is at most its projective dimension. Theorem 5.8 shows that the second inequality cannot be improved. The theorem had been proved by Latch in [18] with a rather restrictive assumption on $\mathbf{C}$. However the result turned out to yield more readily to a theorem in module theory on the projective dimension of an $\boldsymbol{\aleph}_{n}$-related flat module.

7. Hochschild dimension. If $\mathscr{Q}$ is an abelian category with exact products and $\mathbf{C}$ is a small category, then the evaluation functors $\mathbb{Q}^{C} \rightarrow \mathbb{Q}$ all have exact right adjoints, and consequently by Lemma 1.2 we know

$$
\sup _{p} \operatorname{pd} D(p) \leqslant \operatorname{pd} D
$$

for any $D \in \mathbb{Q}^{C}$. The question then arises as to how much the right side of (1) can exceed the left side. This gives rise to the notion of Hochschild dimension, which we shall now discuss.

If $K$ is a commutative ring, then a $K$-category is a category $\mathbb{Q}$ equipped with a $K$-module structure on each of its hom sets $\mathcal{Q}\left(A, A^{\prime}\right)$ such that composition is $K$-bilinear. It is equivalent to be given an additive category $\mathbb{Q}$ together with a ring homomorphism $K \rightarrow C(\mathbb{Q})$. A one object $K$-category is just an associative $K$-algebra, and so we shall call a small $K$-category a $K$-algebroid. If $\mathcal{C}$ is a $K$-algebroid, then Mod $\mathcal{C}$ is a $K$-category in the obvious way. If $\mathscr{Q}$ and $\mathscr{B}$ are $K$-categories, then a functor $F: \mathbb{Q} \rightarrow \mathscr{B}$ is a $K$-functor if $\mathscr{Q}\left(A, A^{\prime}\right) \rightarrow \mathscr{B}\left(F A, F A^{\prime}\right)$ is a $K$-module homomorphism for all $A, A^{\prime}$. If $\mathbb{Q}$ is a $K$-category and $\mathcal{C}$ is a $K$-algebroid, then $\mathfrak{Q}^{\mathfrak{E}}$ denotes the category of all $K$-functors $\mathcal{C} \rightarrow \mathbb{Q}$. The category Mod $\mathcal{C}$ can be identified with the category $(\operatorname{Mod} K)^{e}$. The tensor product $\mathcal{C} \otimes_{K}$ D of $K$-algebroids can be formed in the obvious way so as to yield an isomorphism

$$
\mathbb{Q}^{C \otimes_{K} \mathscr{D}} \simeq\left(\mathbb{Q}^{e}\right)^{\mathscr{D}} \text {. }
$$

In particular we have $\operatorname{Mod} C \otimes_{K} \mathscr{D} \simeq(\operatorname{Mod} C)^{\mathscr{D}}$.

If $\mathcal{C}$ is a $K$-algebroid, then the two variable hom functor $\mathcal{C}($, , can be

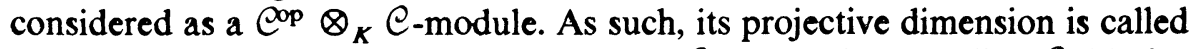
the Hochschild dimension of the $K$-algebroid $\mathcal{C}$, and is denoted $\operatorname{dim}_{K} \mathcal{C}$. Notice that $\mathcal{C}^{\mathrm{op}} \otimes_{K} \mathcal{C}$ is isomorphic to its own opposite, from which it follows that $\operatorname{dim}_{K} \mathcal{C}=\operatorname{dim}_{K}$ eop. If $\mathcal{C}$ and $\mathscr{D}$ are $K$-algebroids, then they are Morita equivalent if Mod $C$ and Mod $\mathscr{D}$ are equivalent via a $K$-functor. It is not hard to see in this case that $\operatorname{dim}_{K} \mathrm{e}=\operatorname{dim}_{K} \mathscr{D}$. A $K$-algebroid $\bigodot$ is $K$-projective if $\mathcal{C}(p, q)$ is a projective $K$-module for all $p, q$.

THEOREM 7.1. Let $\mathcal{C}$ be a $K$-projective algebroid, and let $\mathfrak{Q}$ be an abelian $K$-category with exact coproducts. Then

$$
\text { pd } D<\operatorname{dim}_{K} \mathcal{C}+\sup _{p} \operatorname{pd} D(p)
$$

for all $D \in \mathbb{Q}^{\mathfrak{e}}$.

The proof is in $\left[24\right.$, p. 60]. To see that $\operatorname{dim}_{K} e$ cannot be replaced by anything smaller in the inequality, let $\mathbb{Q}=\operatorname{Mod} \mathcal{C}^{\text {ep }}$, and let $D=\mathcal{C}($, ). Then the values of $D$ are the representable modules $\mathcal{C}(, p)$, which are projective, and consequently the inequality in this case is an equality. 
If $\mathbf{C}$ is a small category, then $K \mathbf{C}$ is a $K$-algebroid. We define the $K$-Hochschild dimension of $\mathbf{C}$ to be $\operatorname{dim}_{K} K \mathbf{C}$, which we denote more simply by $\operatorname{dim}_{K} \mathbf{C}(\operatorname{dim} \mathbf{C}$ when $K=\mathbf{Z})$. The four observations on cohomological dimension at the beginning of $\$ 5$ are also valid for Hochschild dimension. In particular, $\operatorname{dim}_{K} C \leqslant \operatorname{dim} \mathbf{C}$ for all commutative rings $K$. If $\mathscr{Q}$ is a $K$-category, then $\mathbb{Q}^{K C}=\mathbb{Q}^{\mathrm{C}}$. Since $K \mathrm{C}$ is $K$-projective (in fact, $K$-free), Theorem 7.1 becomes:

COROLlary 7.2. If $\mathcal{Q}$ is an abelian $K$-category with exact coproducts, then

$$
\text { pd } D \leqslant \operatorname{dim}_{K} \mathbf{C}+\sup _{p} \operatorname{pd} D(p)
$$

for all $D \in \mathbb{Q}^{\mathbf{C}}$.

In particular, taking $D=\Delta K \in \operatorname{Mod} K \mathbf{C}$, we get:

Corollary 7.3. $\operatorname{cd}_{K} \mathbf{C} \leqslant \operatorname{dim}_{K} \mathbf{C}$.

For groups this inequality is an equality [5, p. 195]. However we know that this can't be true in general since $\mathrm{cd}_{K} \mathbf{C} \neq \mathrm{cd}_{K} \mathbf{C}^{\text {op }}$.

Suppose $\operatorname{dim} \mathbf{C}=0$. Then by Corollary 7.3 we have $\operatorname{cd} \mathbf{C}=0$, so if we assume $\mathbf{C}$ is connected and idempotents split, then by Theorem 5.1 we know $\mathbf{C}$ has an initial object $p$. But $\operatorname{dim} \mathbf{C}=\operatorname{dim} \mathbf{C}^{\text {op }}$, so $\mathbf{C}$ has a terminal object $q$. If $\mathbf{C}(p, q)$ were empty, then $\mathbf{C}$ would contain 2 as a retract. But from Corollary 7.2 and the example following the dimension raising lemma we know $\operatorname{dim} \mathbf{2} \geqslant 1$. Hence $\operatorname{dim} \mathbf{C} \geqslant 1$, a contradiction. Thus $\mathbf{C}(q, p)$ is nonempty, and so $\mathbf{C}$ has a zero object. Therefore if we assume that $\mathbf{C}$ is idempotent free (more precisely, the only idempotents are identities), we obtain:

Proposition 7.4. If $\mathbf{C}$ is idempotent free, then $\operatorname{dim} \mathbf{C}=0$ if and only if $\mathbf{C}$ is equivalent to a discrete category.

I don't know what happens when $\mathrm{C}$ has nonidentity idempotents. One could conjecture that $\operatorname{dim} \mathbf{C}=0$ if and only if $\mathbf{Z C}$ is Morita equivalent to ZD for some discrete category $\mathbf{D}$.

Let us turn to Hochschild dimension one. By the inequality (2) of \$2, we have $\operatorname{dim} \mathbf{C} \leqslant 1$ whenever $\mathbf{C}$ is a category of fractions of a free category. I expect that if $\mathbf{C}$ is idempotent free, then the converse holds. This is true when $\mathbf{C}$ is a group by the Stallings-Swan theorem. Another instance where it is true is given by the following theorem.

THEOREM 7.5. If $\mathbf{C}$ is a small category in which the only endomorphisms are identities, then $\operatorname{dim} \mathbf{C} \leqslant 1$ if and only if $\mathbf{C}$ is free.

This was proved for posets in [24]. The more general theorem will appear in Cheng [9].

The idempotent free categories of fractions of free categories are precisely the bridge categories of the generalized syzygy theorem. These categories have the following strong property, which allows us to give examples of categories of arbitrary Hochschild dimension.

THEOREM 7.6 [24, p. 119]. Let $\mathrm{C}$ be a bridge category which is not equivalent 
to a discrete category. Then for any $K$-algebroid $\subseteq$ we have

$$
\operatorname{dim}_{K} e \bigotimes_{K} K \mathbf{C}=1+\operatorname{dim}_{K} \mathrm{e} \text {. }
$$

As for categories $\mathbf{C}$ of Hochschild dimension $\leqslant 2$, a large class of them was described in terms of generators and relations for $\mathbf{C}$ in [24, p. 110]. For a certain class of categories the description turned out to be necessary as well as sufficient [24, p. 125]. This used the extended exact sequence (1) of $\$ 2$ referred to in the remark of that section.

The following is a slight improvement on [24, Corollary 37.6].

THEOREM 7.7. If $\mathbf{C}$ is a totally ordered set whose closed intervals all have cardinal number at most $\aleph_{n}$, then

$$
\operatorname{dim} C \leqslant n+2 .
$$

It should be possible to determine the precise Hochschild dimension of any totally ordered set. Note that the ordered set of integers has dimension one since it is free. The rationals have dimension $\leqslant 2$ by Theorem 7.7 and $\geqslant 2$ by Theorem 7.5. The dimension of the ordered set of real numbers probably depends on the continuum hypothesis.

8. Free ideal ringoids. A ringoid $C$ has invariant basis number (ibn) if any two bases for a $\mathcal{C}$-module have the same number of elements. This can obviously be formulated in terms of the nonexistence of a pair of nonsquare matrices whose product in either order is defined and is the identity. It follows by passing to transposes that if $e$ has ibn, then so does $e^{o p}$. It also follows that if there exists a map (additive functor) $e \rightarrow \mathscr{D}$ of ringoids and if (1) has ibn, then so does $\mathcal{C}$. In particular, if $R$ is a ring with ibn and $\mathbf{C}$ is any small category, then $R \mathrm{C}$ has ibn, for we always have the map $R \mathrm{C} \rightarrow R$ which sums coefficients. Note that if $\mathcal{C}$ has ibn, then it can have no zero object, for otherwise a free module on one generator would be isomorphic to the free module on no generators. Likewise $e$ can have no product of the form $C \oplus C^{\prime}$ since otherwise a free module on one generator would be isomorphic to a free module on two generators.

If $\mathcal{C}$ has ibn and if every left ideal is free, then $\mathcal{C}$ is called a free left ideal ringoid, or more briefly, a left firoid. $\mathcal{C}$ is a right firoid if $\mathcal{C}^{\circ o}$ is a left firoid, and is a two sided firoid if it is both left and right. Using the ibn, it is easy to see that a left firoid is a domain (that is, $\beta \alpha=0 \Rightarrow \alpha=0$ or $\beta=0$ ). In particular if $R \mathbf{C}$ is a left firoid, then $R$ is a domain and $\mathbf{C}$ is cancellative. Another easy consequence of the ibn is that if two principal left ideals in a left firoid have nonzero intersection, then their sum is principal. When there is just one object we say fir instead of firoid. The remark of $\$ 4$ gives examples of left firoids which are not right firoids.

A complete description of two sided firoids of the form $R \mathrm{C}$ has been given by Roman Wong [30].

THEOREM 8.1 (WONG). $R \mathbf{C}$ is a two sided firoid if and only if $\mathbf{C}$ is a bridge category and $R$ is a division ring, or $\mathbf{C}$ is equivalent to a discrete category and $R$ is a fir.

COROLLARY 8.2. If $\mathbf{C}$ is a nontrivial monoid, then $R \mathbf{C}$ is a two sided fir if and 
only if $R$ is a division ring and $\mathrm{C}$ is the free product of a free monoid and a free group.

The "if" direction of the corollary is a theorem of P. M. Cohn [10] who obtained it as a consequence of a general result on free products of firs. However Wong gave a direct proof. For the "only if" he used another result of Cohn, suitably generalized to categories, characterizing those monoids which are free products of a free monoid and some group. One then invokes the Stallings and Swan theorem to show that the group must actually be free. Now if $\mathbf{C}$ is not equivalent to a discrete category, then once one has shown that it is a bridge category, one knows by the generalized syzygy theorem that $\mathrm{gl} \operatorname{dim} R \mathrm{C}=1+\mathrm{gl} \operatorname{dim} R$. But a left firoid is hereditary, and so $\mathrm{gl} \operatorname{dim} R \mathrm{C}$ $=1$. Hence $\mathrm{gl} \operatorname{dim} R=0$, that is, $R$ is semisimple, and so being a domain, it must be a division ring.

9. Projective free ringoids. A ringoid is projective free if every projective

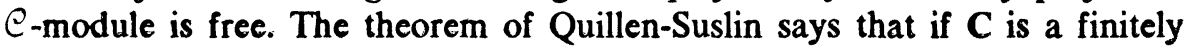
generated free commutative monoid and $R$ is a pid, then $R C$ is projective free. Examples in [2] show that $R$ cannot be replaced by a general projective free ring.

Any left firoid is projective free. For a left firoid is hereditary, and consequently every projective module is a coproduct of left ideals [5, p. 13]. In particular, by Theorem 8.1 we know that if $K$ is a field and $C$ is a bridge category, then $K \mathrm{C}$ is projective free. It would be interesting to know if $K$ can be replaced by a pid. Of course we know that $K$ can't be replaced by any projective free ring because of the example of the polynomial ring. In [3], Bass showed that if $\mathbf{C}$ is a free monoid or free group and $R$ is a pid, then finitely generated projective modules over $R \mathrm{C}$ are free.

A ringoid $\mathcal{C}$ is local if every representable $\mathcal{C}(p$,$) has a unique maximal left$ ideal. As in the case of rings, $\mathcal{C}$ is local if and only if $\mathcal{C}^{\circ}$ is local. The proof of Kaplansky [17] for rings generalizes to give the following theorem.

THEOREM 9.1. A local ringoid is projective free.

If $K$ is a division ring and $\mathrm{C}$ is a poset, then it is obvious that $K \mathrm{C}$ is local. Thus:

COROLlary 9.2. If $K$ is a division ring and $\mathrm{C}$ is a poset, then $K \mathrm{C}$ is projective free.

If $\mathrm{C}$ is assumed to have the dcc, then the division ring $K$ of the corollary can be replaced by any projective free ring:

THEOREM 9.3 [7]. If $R$ is a projective free ring and $\mathrm{C}$ is a poset with dcc, then $R \mathrm{C}$ is projective free.

Except for Corollary 9.2, we have no idea what happens to Theorem 9.3 when the dcc is removed. For example, if $\mathbf{C}$ is the poset of negative integers, is $\mathbf{Z C}$ projective free? Of course this example may be special, since here $\mathbf{C}$ is free as a category. In any case, a single example of a non dcc poset $\mathbf{C}$ and a projective free, nondivision ring $R$ such that $R \mathrm{C}$ either is or is not projective free would be illuminating. 
10. Perfect ringoids. The Jacobson radical of a ringoid $C$ can be defined as the two sided ideal $J$ such that $J(p$,$) is the intersection of the maximal left$ ideals in $\mathcal{C}(p$,$) , or equivalently, such that J(, q)$ is the intersection of the maximal right ideals in $\mathcal{C}(, q)$. The usual module theoretic facts relating to the Jacobson radical, including Nakayama's lemma, are valid. In particular, $\mathcal{C}$ is semisimple if and only if it is artinian and $J=0$.

An ideal $I$ of $C$ is $T$-nilpotent if for every sequence $\alpha_{1}, \alpha_{2}, \ldots$ of composable elements of $I$ we have $\alpha_{1} \alpha_{2} \cdots \alpha_{n}=0$ for some $n$. A ringoid is perfect if every module has a projective cover (defined dually to injective hull). One can then repeat Bass' proof [1] of the following theorem.

THEOREM 10.1 (BASS). The following conditions are equivalent on a ringoid $\mathcal{C}$.

(a) $\mathcal{C}$ is perfect.

(b) Every flat C-module is projective.

(c) $C$ has the dcc on principal right ideals.

(d) $\mathcal{C}(p, p)$ contains no infinite set of orthogonal idempotents for each $p$, and every nonzero eop-module contains a simple submodule.

(e) $\mathcal{C} / J$ is semisimple and $J$ is $T$-nilpotent.

If $\mathrm{C}$ is a poset and $K$ is a division ring, then the Jacobson radical of $K \mathrm{C}$ is easily seen to be given by $J(p, q)=K$ if $p<q$ and 0 otherwise. Thus $K \mathrm{C} / J$ is a disjoint union of division rings, and so is semisimple. Moreover we see that $J$ is $T$-nilpotent if and only if $\mathbf{C}$ has the dcc. By virtue of condition (e) of the theorem, this provides examples of perfect ringoids whose opposites are not perfect.

Bass asked if the dcc on principal right ideals implies the dcc on finitely generated right ideals. This question was answered with a vengeance by Björk [4], who showed that in any module the dcc on cyclic submodules implies the dcc on finitely generated submodules. However I would like to show why it is, granting that Bass' theorem applies to ringoids, that Bass had essentially answered his own question. First observe that finitely generated means cyclic in a ringoid with finite products. Now any ringoid $e$ is contained as a full subcategory in a ringoid $\hat{e}$ with finite products in such a way that every object of $\hat{C}$ is a finite product of objects of $\mathcal{C}$. (One just takes $\hat{\mathcal{C}}$ to be the ringoid whose morphisms are finite matrices of morphisms of $\mathcal{C}$.) The latter property guarantees that the restriction functor $\operatorname{Mod} \hat{\mathrm{C}} \rightarrow \operatorname{Mod} \mathcal{C}$ is an equivalence of categories. Since perfection is a Morita invariant, it follows that $\hat{\mathcal{C}}$ is perfect if and only if $\mathcal{C}$ is. But Mod $\hat{\mathcal{C}}^{\mathrm{op}} \rightarrow \operatorname{Mod} \mathcal{C}^{\mathrm{op}}$ is also an equivalence of categories, and any representable in Mod eop comes from one in Mod $\hat{C}^{\text {op }}$. Thus a descending chain of finitely generated right ideals in $\mathcal{C}$ gives rise to a descending chain of principal right ideals in $\mathcal{C}$, which must terminate if $\mathcal{C}$, hence $\hat{\mathcal{C}}$, is perfect.

11. The universal counterexample. Most counterexamples in this paper, in particular all examples showing that a notion is not left-right symmetric, are given by the ringoid $K \mathbf{C}$ where $K$ is a field and $\mathrm{C}$ is an appropriate ordinal number considered as an ordered set, hence as a category. Of course similar examples exist in rings, but there one usually has to be more clever. For example, there are left principal ideal domains with arbitrarily large right 
global dimension (Jategaonkar [16]). In particular, these provide examples of left firs which are not right firs. (See also Cohn [11].) Also there are examples of Bass [1] exhibiting perfect rings whose opposites are not perfect.

We shall conclude with one additional use of the above example $K C$. First, if $\mathbf{C}$ is a totally ordered set and $K$ is a field, then it is easy to see that $K \mathbf{C}$ is noetherian if and only if $\mathbf{C}$ does not contain $(\omega+1)^{\text {op }}$ as a subcategory, and $K \mathrm{C}$ is artinian if and only if $\mathbf{C}$ does not contain $\omega$ as a subcategory. This provides examples of ringoids which are left but not right noetherian (artinian). But more important, it also provides examples of ringoids which are left artinian but not left noetherian. This, then, serves as a word of caution concerning our principal theme, namely, that theorems about rings are really theorems about ringoids.

\section{REFERENCES}

1. H. Bass, Finitistic dimension and a homological generalization of semi-primary rings, Trans. Amer. Math. Soc. 95 (1960), 466-488.

2. __ Torsion free and projective modules, Trans. Amer. Math. Soc. 102 (1962), 319-327.

3. __ Projective modules over free groups are free, J. Algebra 1 (1964), 367-373.

4. J.-E. Björk, Rings satisfying a minimum condition on principal ideals, J. Reine Angew. Math. 236 (1969), 112-119. 1956.

5. H. Cartan and S. Eilenberg, Homological algebra, Princeton Univ. Press, Princeton, N. J.,

6. C. Cheng and B. Mitchell, Flatness and projectivity of modules that come from C-sets, Algebra, Topology, and Category Theory, Academic Press, New York, 1976, pp. 33-44.

7. $ـ$ DCC posets of cohomological dimension one, J. Pure Appl. Algebra (to appear).

8. C. Cheng, Finite partially ordered set of cohomological dimension one, J. Algebra 40 (1976), 340-347.

9. __ Deltas of Hochschild dimension one, Proc. Amer. Math. Soc. 67 (1977), 221-223.

10. P. M. Cohn, Free ideal rings, J. Algebra 1 (1964), 47-69.

11. __ Free rings and their relations, Academic Press, New York, 1971.

12. S. Eilenberg, A. Rosenberg, and D. Zelinsky, On the dimension of modules and algebras.

VIII, Nagoya Math. J. 12 (1957), 71-93.

13. G. Hochschild, Note on relative homological dimension, Nagoya Math J. 13 (1958), 89-94.

14. J. Isbell, Exact colimits. I, Ann. of Math. (2) 100 (1974), 633-637.

15. J. Isbell and B. Mitchell, Exact colimits and fixed points, Trans. Amer. Math. Soc. 220 (1976), 289-298.

16. A. V. Jategaonkar, A counterexample in ring theory and homological algebra, J. Algebra 12 (1969), 418-440.

17. I. Kaplansky, Projective modules, Ann. of Math. (2) 68 (1958), 372-377.

18. D. Latch, On derived functors of limit, Trans. Amer. Math. Soc. 181 (1973), 155-164.

19. D. Latch and B. Mitchell, On the difference between cohomological and homological dimension, J. Pure Appl. Algebra 5 (1974), 333-343.

20. O. Laudal, Note on the projective limit on small categories, Proc. Amer. Math. Soc. 33 (1972), 307-309.

21. D. Lazard, Autour de la platitude, Bull. Soc. Math. France 97 (1969), 81-128.

22. R. C. Lyndon, Cohomology theory of groups with a single defining relation, Ann. of Math. (2) 52 (1950), 650-665.

23. B. Mitchell, On the dimension of objects and categories. I, II, J. Algebra 9 (1968), 314-368.

24. , Rings with several objects, Advances in Math. 8 (1972), 1-161.

25. __ The dominion of Isbell, Trans. Amer. Math. Soc. 167 (1972), 319-331.

26. __ The cohomological dimension of a directed set, Canad. J. Math. 25 (1973), 233-238. 
27. B. Osofsky, Homological dimension and the continuum hypothesis, Trans. Amer. Math. Soc. 132 (1968), 217-230.

28. J. R. Stallings, On torsion free groups with infinitely many ends, Ann. of Math. (2) 88 (1968), 312-334.

29. R. G. Swan, Groups of cohomological dimension one, J. Algebra 12 (1969), 585-610.

30. R. Wong, Monoid rings which are free ideal rings, J. Algebra (to appear).

Department of Mathematics, Rutgers University, New Brunswick, New Jersey 08903 